\title{
Convenient synthesis of new functionalized cyclopropanes from monoterpenic olefines
}

\author{
Hossni Ziyat, ${ }^{a}$ My Youssef Ait Itto, ${ }^{a}$ Mustapha Ait Ali, ${ }^{a *}$ Abdelkhalek Riahi, \\ Abdellah Karim, ${ }^{a}$ and Jean-Claude Daran ${ }^{\mathrm{c}}$ \\ ${ }^{a}$ Departement de Chimie Faculte des Siences-Semlalia, BP 2390 Marrakech, Morocco \\ ${ }^{b}$ UMR 6519 "Réactions sélectives et applications", Université de Reims Champagne-Ardenne, \\ Faculté des Sciences, Moulin de la Housse, BP 1039 - 51687 Reims CEDEX 2, France \\ ${ }^{c}$ Laboratoire de Chimie de Coordination, CNRS UPR8241, 205 Route de Narbonne, 31077 \\ Toulouse CEDEX, France \\ E-mail : aitali@ucam.ac.ma, $\underline{\text { m aitali@hotmail.com }}$
}

\begin{abstract}
Five new cyclopropyl-ketoacids were prepared in good yields from monoterpenic olefines using solid-liquid phase transfer catalysis (SL-PTC) dichlorocyclopropanation reaction followed by oxidative cleavage with $\mathrm{RuCl}_{3}-\mathrm{NaIO}_{4}$ system. The nonchlorinated cyclopropanes were obtained by sodium/methanol reduction of the corresponding gem-dichlorocyclopropanes.
\end{abstract}

Keywords : monoterpenes, cyclopropyl-ketoacids, phase transfer catalysis, oxidative cleavage

\section{Introduction}

The enormous importance of functionalized cyclopropanes, in various scientific fields, lies in their diverse biological activity ${ }^{1}$ and their usefulness as valuable building blocks in organic synthesis. ${ }^{2}$ Recently ${ }^{3-5}$, we have reported the synthesis of new functionalized cyclopropanes from terpenic olefins. This synthesis involves a periselective gem-dihalocyclopropanation reaction (under SL-PTC conditions) ${ }^{6}$ of one of the terpenic olefine double bounds followed by an oxidative cleavage of the other, using $\mathrm{RuCl}_{3}-\mathrm{NaIO}_{4}$ catalytic system. ${ }^{7}$ This facile and convenient methodology has been adapted for the preparation of five new cyclopropyl-ketoacids which are reported herein. 


\section{Results and discussion}

Three monoterpenes were selected as starting materials : limonene 1, terpinolene $\mathbf{2}$ and $\gamma$ terpinene $\mathbf{3}$. We notice here that for $\mathbf{1}$ and $\mathbf{2}$, our strategy consists in a dichlorocyclopropanation of the exocyclic double bound (Scheme 1), before the oxidative cleavage of the internal one.

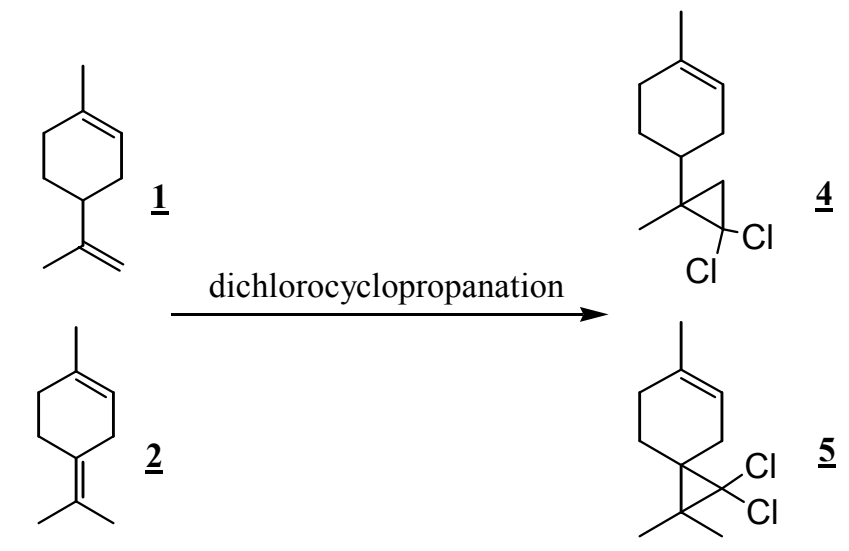

\section{Scheme 1}

The dichlorocyclopropanation reaction was achieved using phase-transfer catalysis technique. The primary advantages of using phase-transfer catalysis are to obtain a large conversion, high reaction rate and selectivity at moderate reaction conditions. ${ }^{8-10}$ It is interesting to note that powdered $\mathrm{KOH}$ or $\mathrm{NaOH} / \mathrm{BTEAC} / \mathrm{CHCl}_{3}$ has been suggested as a very effective $\mathrm{CCl}_{2}$ precursor. ${ }^{11} \mathrm{~A}$ comparison with the original Makosza method showed similar yields, but the process with solid base was proved to be faster. ${ }^{10-11}$

Earlier $^{12}$, Sirovski and al. have published the work discussing various models of phasetransfer catalysed reactions in the different solid/liquid systems. It was shown that Makosza reaction in this system is hindered by the formation of the crust of $\mathrm{NaCl}$.

A suggestion was put forward that phase-transfer catalysed reactions in these systems closely resemble to the so-called topochemical processes (solid/fluid) described elsewhere. ${ }^{13}$

For this reason, we have studied the cyclopropanation reaction of limonene $\mathbf{1}$, terpinolene $\mathbf{2}$ and $\gamma$-terpinene $\mathbf{3}$ under controlled solid/liquid phase transfer catalysis conditions. Dichlorocarbene was used as the carbene component. It was generated by the adapted method to terpenic olefins ${ }^{14}$, i.e., reaction of powdered $\mathrm{NaOH}$ with chloroform at room temperature in the presence of benzyltriethylammonium chloride (BTEAC) as the phase transfer catalyst. We found that 2 and 3 afforded the desired gem-dichlorocyclopropanes 5 (71\%) and $\mathbf{6}(74 \%)$, respectively, while limonene 1 led to the nondesired product $7^{15}(62 \%)$ as a mixture of cis/trans stereoisomers in which the internal double bound was affected (Scheme 2). We notice here, that despite the variation of the experimental parameters such as phase transfer catalysts, catalyst amount, 
sodium hydroxide concentration and temperature, the dichlorocarbene addition reaction to limonene $\mathbf{1}$ always gives the nondesired product $7^{15}$.
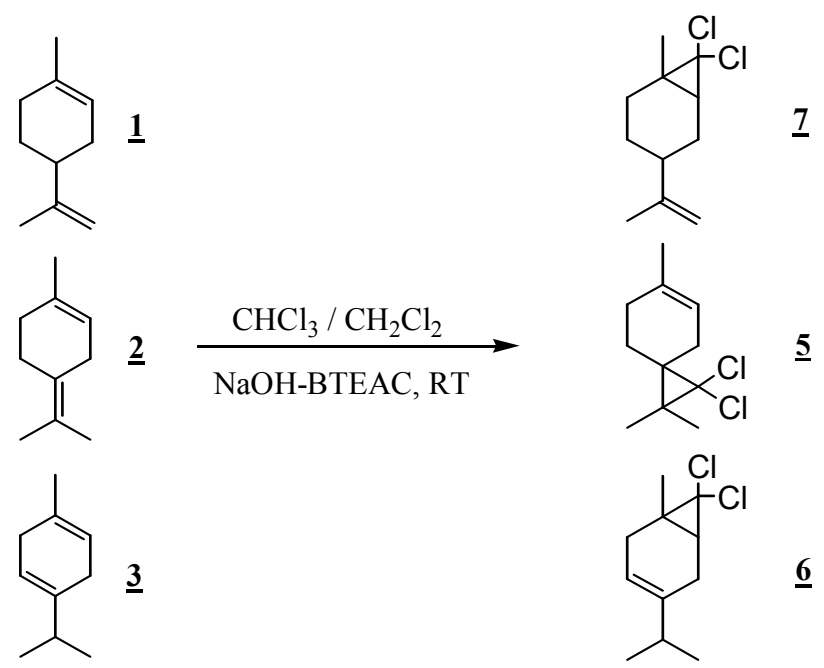

\section{Scheme 2}

In order to direct the dichlorocyclopropanation reaction towards the exocyclic double bound, 1 was first epoxidized under aerobic epoxidation conditions described recently ${ }^{16}$. The resulting epoxylimonene $\mathbf{8}^{16}$, obtained as cis/trans (40/60) diastereomeric mixture in $91 \%$ yield, was then treated with dichlorocarbene (generated in-situ under the same conditions described above) to provide the diclorocyclopropane $9^{17}$ in $90 \%$ yield, as a mixture of four stereoisomers (two faces of the double bond in $\mathbf{8}$ are likely not sterically equivalent) (Scheme 3).

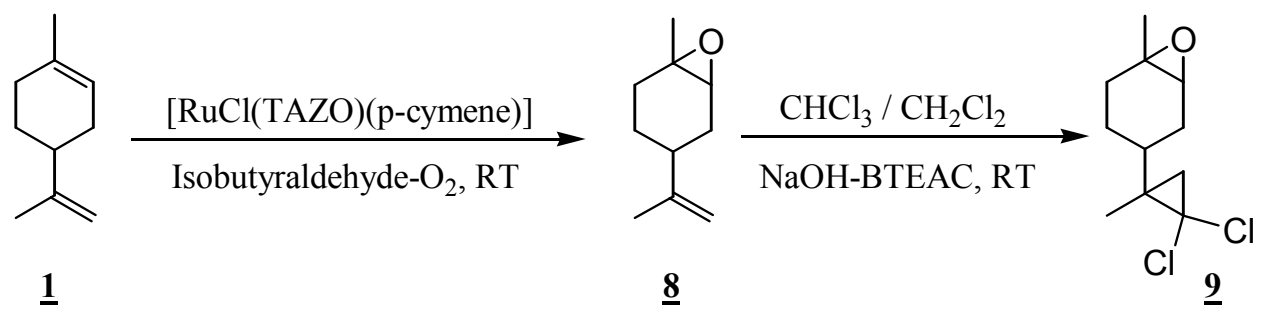

\section{Scheme 3}

All the obtained products 5-9 were fully characterised by their mass and NMR spectroscopic data.

In order to obtain the nonchlorinated cyclopropanes, we reduced compounds 5, $\mathbf{6}$ and 9 using metallic sodium in methanol. The reaction afforded, in excellent yields, the corresponding cyclopropanes $10(92 \%), \mathbf{1 1}(95 \%)$ and $\mathbf{1 2}(97 \%)^{17}$ respectively (Scheme 4). The compound $\mathbf{1 2}$ was also isolated as a cis/trans (31/69) diastereomeric mixture. 
Before oxidation of obtained cyclopropanes 10, 11 and 12, we have first checked the oxidative cleavage reaction on compound $\mathbf{1 2}$ in order to optimize the reaction conditions. Table 1 summarises some representative results obtained with 12 using freshly prepared $\mathrm{RuO}_{4}$ as catalyst and $\mathrm{NaIO}_{4}$ (stoichoimetric) as oxidant.

Table 1: Oxidative cleavage of $\mathbf{1 2}$ using the system $\mathrm{RuCl}_{3}-\mathrm{NaIO}_{4}$.

\begin{tabular}{|c|c|c|c|c|}
\hline Entry & $\mathrm{S} \mathrm{C}^{b}$ & $\mathrm{NaIO}_{4}$ (eq. number) & Time $(\mathrm{h})$ & $\mathbf{1 5}$ yield (\%) \\
\hline 1 & 50 & 10 & 30 & 87 \\
2 & 50 & 8 & 30 & 85 \\
3 & 50 & 4 & 62 & 42 \\
4 & 50 & 2 & 62 & 05 \\
5 & 100 & 8 & 48 & 71 \\
6 & 500 & 8 & 48 & 10 \\
\hline
\end{tabular}

${ }^{a}$ Conditions: $\mathbf{1 2}=3.1 \mathrm{mmol} ; \mathrm{H}_{2} \mathrm{O}=6 \mathrm{~mL} ; \mathrm{CH}_{3} \mathrm{CN}=4 \mathrm{~mL} ; \mathrm{CCl}_{4}=4 \mathrm{~mL}$; Temperature $=20^{\circ} \mathrm{C}$;

${ }^{b} \mathrm{~S} / \mathrm{C}=$ substrate/catalyst.

Compound 12 was readily cleaved to the corresponding ketoacid 15 with high yield (Table 1 entry 1-2) by quenching the reaction after 30 hours (Scheme 4).

An increase of the substrate/catalyst ratio (100 instead 50) led as expected to a slower reaction but interestingly high yield into the acid. With a substrat/catalyst ratio 500 , only $10 \%$ of ketoacid 15 were obtained after 48 hours (entry 6). On the other hand a decrease in the $\mathrm{NaIO}_{4} /$ substrate ratio had a very detrimental effect on conversion (entry 4). The best result $(85 \%$ yield) was obtained at room temperature after 30 hours with 8 equivalent of $\mathrm{NaIO}_{4}$ and substrate/catalyst ratio 50 (entry 2).

Under optimised conditions (8 equivalent of $\mathrm{NaIO}_{4}$, substrate/catalyst ratio 50, room temperature), oxidative cleavage of cyclopropanes 10 and 11 with $\mathrm{RuCl}_{3}-\mathrm{NaIO}_{4}$ system afforded quantitatively the corresponding cyclopropyl-ketoacids 13 (81\%) and $\mathbf{1 4}$ (78\%) (Scheme 4). We notice here that the stereochemistry of $\mathbf{1 4}$ at the cyclopropane must be $\mathrm{Z}$ since it originates from 11.

The spectroscopic data of the three newly prepared cyclopropyl-ketoacids 13, 14 and 15 are consistent with the assigned structures, mainly characterized by ${ }^{1} \mathrm{H}$ NMR spectroscopy; the signals for cyclopropanic protons are ranged between 0.1-0.6 ppm and the signals for ethylenic protons disappeared. Their ${ }^{13} \mathrm{C}$ NMR spectra reveal carbonyl groups resonance at about 209-213 ppm (ketone carbonyl group) and 178-179 ppm (acid carbonyl group). 


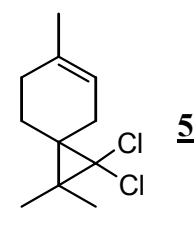<smiles>CC1=CCC(C(C)(C)C)CC1</smiles>

$\underline{10}$<smiles>CC(=O)CCC1(CC(=O)O)CC1(C)C</smiles><smiles>CC(C)C1=CCC2(Cl)CC1C2(Cl)Cl</smiles>

$\underline{\mathbf{6}} \underset{\mathrm{NT} / \mathrm{CH}_{3} \mathrm{OH}}{\longrightarrow}$<smiles>CC(C)C1=CCC2(C)CC1C2</smiles>

$\underline{11}$<smiles>CC(C)CCCCCOC(=O)CC1CC1CC(=O)C(C)C</smiles><smiles></smiles><smiles>CC1(C)CCCC(C)(C)O1</smiles><smiles>CC(=O)CCC(CC(=O)O)C1(C)CC1</smiles>

\section{Scheme 4}

Under identical conditions dichlorocyclopropyl-ketoacids 16 and 17 were similarly prepared by oxidative cleavage of the corresponding precursors 5 and 6 (Scheme 5).

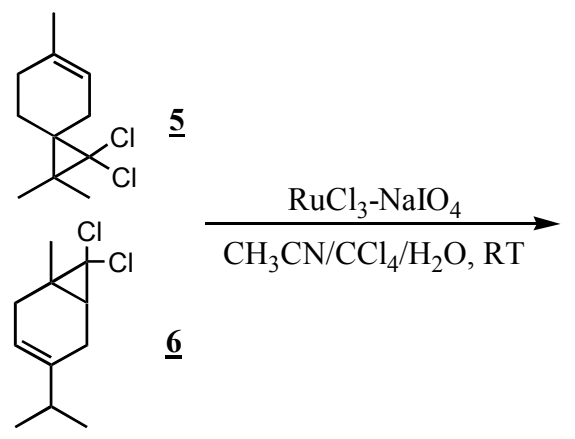<smiles>CC(=O)CCC1(CC(=O)O)C(C)(C)C1(Cl)Cl</smiles><smiles>[3H]C(C)C(=O)CC1C(Cl)(Cl)C1(C)CC(=O)O</smiles>

\section{Scheme 5}

Their spectroscopic data are in full accordance with the attributed structures which were mainly characterized by ${ }^{13} \mathrm{C}$ NMR spectroscopy. The cyclopropanic $\mathrm{CCl}_{2}$ groups appeared at 70 $75 \mathrm{ppm}$, whereas acid and ketone carbonyl groups signals revealed at $176 \mathrm{ppm}$ and 208-212 ppm respectively.

\section{Conclusion}

We have described a simple and convenient synthesis of new cyclopropyl-ketoacids starting from limonene, terpinolene and $\gamma$-terpinene. The nonchlorinated cyclopropyl-ketoacids 13-15 were prepared by a periselective dichlorocyclopropanation reaction (under SL-PTC conditions) 
of the corresponding monoterpenes followed by a sodium/methanol reduction of the gemdichlorocyclopropanes 5, 6 and 9. The oxidative cleavage of the obtained cyclopropanes 10-12 with system $\mathrm{RuCl}_{3}-\mathrm{NaIO}_{4}$, afforded the desired cyclopropyl-ketoacids 13-15 in 53-67\% overall yields. It is worth mentioning that in the case of limonene, we were compelled to epoxidize the internal double bound in order that the ensuing dichlorocyclopropanation reaction would occur at the exocyclic one. The dichlorocyclopropyl-ketoacids $\mathbf{1 6}$ and $\mathbf{1 7}$ were similarly obtained via a direct oxidative cleavage from the corresponding precursors 5 and $\mathbf{6}$ in $56 \%$ and $62 \%$ overall yields, respectively.

\section{Experimental Section}

General. All reagents and solvents were purchased from commercial sources (Aldrich, 112 Acros) and used as received. The reaction mixtures were analysed on a Trace 2000 series chromatograph equipped with an FID detector, using silica capillary columns CPSil5CB (10 m $\times$ $0.33 \mathrm{~mm}$, Chrompack). Column chromatography was performed on silica gel (Merck 60, 220$440 \mathrm{mesh})$. A BP5 (25 m $\times 0.25 \mathrm{~mm})$ capillary column was used for GC/MS coupled analyses with a Saturn 2000 spectrometer. ${ }^{1} \mathrm{H}$ NMR and ${ }^{13} \mathrm{C}$ NMR spectra were recorded on a Bruker Model AVANCE 300 using $\mathrm{CDCl}_{3}$ as the solvent and $\mathrm{SiMe}_{4}$ as the internal standard.

\section{Dichlorocyclopropanation:}

At $0^{\circ} \mathrm{C} 3.00 \mathrm{~g}(75 \mathrm{mmol})$ of sodium hydroxide was added to $6.00 \mathrm{~mL}(74 \mathrm{mmol})$ of chloroform and $0.02 \mathrm{~g}(0.1 \mathrm{mmol})$ of benzyltriethylammonium chloride (BTEAC) in $6 \mathrm{~mL}$ of dichloromethane. The mixture was stirred for $10 \mathrm{~min}$, then $0.86 \mathrm{~g}(6.3 \mathrm{mmol})$ of the monoterpene was added dropwise over a period of $30 \mathrm{~min}$. The mixture was strirred for $8 \mathrm{~h}$ at $25^{\circ} \mathrm{C}$ and then hydrolyzed by addition of $20 \mathrm{~mL}$ of water. The organic layer was separated and the aqueous layer was washed three times with $10 \mathrm{~mL}$ of dichloromethane. The combined organic extracts were dried over $\mathrm{Na}_{2} \mathrm{SO}_{4}$ and concentrated under reduced pressure. The residue was then subjected to purification by silica gel column chromatography (hexane) to afford the corresponding gem-dichlorocyclopropane as colorless oils.

1,1-Dichloro-2,2,6-trimethyl-spiro[2.5]oct-5-ene (5). Yield : 71\%; m/z : 223 (0.55 \%, $\left.\left.\mathrm{MH}+4\rceil^{+}\right) ; 221(3.34 \%, \mathrm{MH}+2\rceil^{\dagger}\right) ; 219\left(5.17 \%, \mathrm{MH}^{\dagger}\right) ;{ }^{1} \mathrm{H} \mathrm{NMR}\left(\mathrm{CDCl}_{3}\right): \delta=1.20(\mathrm{~s}, 3 \mathrm{H})$, $1.30(\mathrm{~s}, 3 \mathrm{H}), 1.50-1.70(\mathrm{~m}, 2 \mathrm{H}), 1.75(\mathrm{~s}, 3 \mathrm{H}), 1.80-2.40(\mathrm{~m}, 4 \mathrm{H}), 5.40(\mathrm{br} \mathrm{s}, 1 \mathrm{H}) ;{ }^{13} \mathrm{C} \mathrm{NMR}$ $\left(\mathrm{CDCl}_{3}\right): \delta=18.9\left(\mathrm{CH}_{3}\right), 19.1\left(\mathrm{CH}_{3}\right), 22.9\left(\mathrm{H}_{2} \mathrm{C} 8\right), 25.7\left(\mathrm{CH}_{3}\right), 28.7\left(\mathrm{H}_{2} \mathrm{C} 7\right), 29.2\left(\mathrm{H}_{2} \mathrm{C} 4\right), 32.8$ (C2), 33.6 (C3), $67.0\left(\mathrm{Cl}_{2} \mathrm{C} 1\right), 119.1(\mathrm{HC} 5), 134.7$ (C6).

7,7-Dichloro-4-isopropyl-1-methyl-bicyclo[4.1.0]hept-3-ene (6). Yield : 74 \%; m/z : 223 (3.25 $\left.\left.\left.\%, \mathrm{MH}+4\rceil^{+}\right) ; 221(20.71 \%, \mathrm{MH}+2\rceil^{+}\right) ; 219(33.72 \%, \mathrm{MH}\rceil^{+}\right) ;{ }^{1} \mathrm{H}$ NMR $\left(\mathrm{CDCl}_{3}\right): \delta=0.98(\mathrm{~d}, \mathrm{~J}$ $=6.9 \mathrm{~Hz}, 6 \mathrm{H}), 1.05(\mathrm{dd}, \mathrm{J}=14.1,7.9 \mathrm{~Hz}, 1 \mathrm{H}), 1.44(\mathrm{~s}, 3 \mathrm{H}), 2.00-2.50(\mathrm{~m}, 5 \mathrm{H}), 5.26(\mathrm{br} \mathrm{s}, 1 \mathrm{H})$;

${ }^{13} \mathrm{C}$ NMR $\left(\mathrm{CDCl}_{3}\right): \delta=20.9\left(\mathrm{H}_{2} \mathrm{C} 5\right), 21.2\left(\mathrm{CH}_{3}\right), 21.3\left(\mathrm{CH}_{3}\right), 24.4\left(\mathrm{CH}_{3}\right), 28.7\left(\mathrm{H}_{2} \mathrm{C} 2\right), 30.8$ $(\mathrm{C} 1), 31.6\left(\mathrm{HC}\left(\mathrm{CH}_{3}\right)_{2}\right), 34.8(\mathrm{HC} 6), 72.2\left(\mathrm{Cl}_{2} \mathrm{C} 7\right), 115.5(\mathrm{HC} 3), 138.7(\mathrm{C} 4)$.

4-(2',2'-Dichloro-1'-methylcyclopropyl)-1-methyl-7-oxabicyclo[4.1.0]heptane (9). Obtained as an inseparable diastereomeric mixture.Yield : $90 \%$; m/z: 239(0.35\%, MH+4 $\left.{ }^{+}\right) ; 237(2.12$ 
$\left.\left.\%, \mathrm{MH}+27^{+}\right) ; 235(3.28 \%, \mathrm{MH}\rceil^{+}\right) ;{ }^{1} \mathrm{H}$ NMR $\left(\mathrm{CDCl}_{3}\right): \delta=1.05 \quad(\mathrm{~s}, 2.1 \mathrm{H}$, major diastereoisomers), $1.06(\mathrm{~s}, 0.9 \mathrm{H}$, minor diastereoisomers $), 0.91-1.20(\mathrm{~m}, 2 \mathrm{H}), 1.23(\mathrm{~s}, 3 \mathrm{H}), 1.28$ 1.63(m, $4 \mathrm{H}), 1.73(\mathrm{~d}, \mathrm{~J}=14.3,0.3 \mathrm{H}$, minor diastereoisomers), $1.76(\mathrm{~d}, \mathrm{~J}=14.2,0.7 \mathrm{H}$, major diastereoisomers), $2.05(\mathrm{~m}, 1 \mathrm{H}), 2.08(\mathrm{~m}, 0.7 \mathrm{H}$, major diastereoisomers $), 2.10(\mathrm{~m}, 0.3 \mathrm{H}$, minor diastereoisomers), $2.93(\mathrm{~d}, \mathrm{~J}=5.2 \mathrm{~Hz}, 0.7 \mathrm{H}$, major diastereoisomers), $2.95(\mathrm{~m}, 0.3 \mathrm{H}$, minor diastereoisomers); ${ }^{13} \mathrm{C} \mathrm{NMR}\left(\mathrm{CDCl}_{3}\right): \delta=15.6$ and $15.7\left(\mathrm{CH}_{3}\right), 22.9$ and $25.4\left(\mathrm{H}_{2} \mathrm{C} 5\right), 23.0$ $\left(\mathrm{CH}_{3}\right), 26.1$ and $27.9\left(\mathrm{H}_{2} \mathrm{C} 3\right), 29.4$ and $30.8\left(\mathrm{H}_{2} \mathrm{C} 3{ }^{\prime}\right), 32.7$ and $32.8\left(\mathrm{H}_{2} \mathrm{C} 6\right), 31.6$ and $\left.33.1(\mathrm{C})^{\prime}\right)$, 36.1 and 40.0 (HC4), 57.4 and $57.7(\mathrm{C} 1), 59.0$ and $60.4(\mathrm{HC} 2), 68.0$ and $68.7\left(\mathrm{Cl}_{2} \mathrm{C} 2{ }^{\prime}\right)$.

Reduction of gem-dichlorocyclopropanes (general procedure). To a cooled $\left(0^{\circ} \mathrm{C}\right)$ solution of $9.2 \mathrm{mmol}$ of the dichlorocyclopropane in $50 \mathrm{~mL}$ of ether were added, simultaneously over a 1hour period, $6 \mathrm{~g}$ ( $260.86 \mathrm{mmol})$ of sodium and $30 \mathrm{ml}$ methanol $(98 \%)$. The mixture was stirred for 3-hours and another portion of $2 \mathrm{~g}(87 \mathrm{mmol})$ of sodium and $20 \mathrm{~mL}$ of methanol $(98 \%)$ were added. Stirring was maintained overnight at room temperature. The mixture was diluted with water and poured into a separatory funnel. The layers were separated and the aqueous phase was extracted with ether. The combined organic layers were dried over anhydrous magnesium sulfate, filtered and concentrated by rotary evaporation. The residue was chromatographied on silica gel column using pentane as the eluent. The corresponding cyclopropanes were obtained as colorless oils.

1,1,6-Trimethyl-spiro[2.5]oct-5-ene (10). Yield : $92 \%$; m/z : $151\left(7.87 \%, \mathrm{MH}^{+}\right)$; ${ }^{1} \mathrm{H}$ NMR $\left(\mathrm{CDCl}_{3}\right): \delta=0.09(\mathrm{~d}, \mathrm{~J}=7.1 \mathrm{~Hz}, 1 \mathrm{H}), 0.14(\mathrm{~d}, \mathrm{~J}=7.1 \mathrm{~Hz}, 1 \mathrm{H}), 1.16(\mathrm{~s}, 3 \mathrm{H}), 1.18(\mathrm{~s}, 3 \mathrm{H}), 1.70(\mathrm{~s}$, $3 \mathrm{H}), 1.91(\mathrm{~m}, 2 \mathrm{H}), 2.10(\mathrm{~m}, 2 \mathrm{H}), 2.33(\mathrm{~m}, 2 \mathrm{H}), 5.15($ br s, $1 \mathrm{H}) ;{ }^{13} \mathrm{C} \mathrm{NMR}\left(\mathrm{CDCl}_{3}\right): \delta=17.2$ (C1), $19.0\left(\mathrm{CH}_{3}\right), 21.9\left(\mathrm{CH}_{3}\right), 23.6\left(\mathrm{CH}_{3}\right), 25.8\left(\mathrm{H}_{2} \mathrm{C} 2\right), 28.1\left(\mathrm{H}_{2} \mathrm{C} 7\right), 30.1\left(\mathrm{H}_{2} \mathrm{C} 4\right), 32.1\left(\mathrm{H}_{2} \mathrm{C} 8\right)$, 37.2 (C3), 121.3 (HC5), 134.3 (C6).

4-Isopropyl-1-methyl-bicyclo[4.1.0]hept-3-ene (11). Yield : $95 \%$; m/z : $151\left(56.56 \%\right.$, $\left.\mathrm{MH}^{+}\right)$; ${ }^{1} \mathrm{H}$ NMR $\left(\mathrm{CDCl}_{3}\right): \delta=0.18(\mathrm{~m}, 2 \mathrm{H}), 0.80(\mathrm{~m}, 1 \mathrm{H}), 0.95(\mathrm{~s}, 3 \mathrm{H}), 0.97(\mathrm{~s}, 3 \mathrm{H}), 1.09(\mathrm{~s}, 3 \mathrm{H}), 1.31$ $(\mathrm{m}, 2 \mathrm{H}), 2.25(\mathrm{~m}, 2 \mathrm{H}), 2.31(\mathrm{~m}, 1 \mathrm{H}), 5.22(\mathrm{br} \mathrm{s}, 1 \mathrm{H}) ;{ }^{13} \mathrm{C} \mathrm{NMR}\left(\mathrm{CDCl}_{3}\right): \delta=13.1\left(\mathrm{H}_{2} \mathrm{C} 7\right), 14.8$ (HC6), $18.9\left(\mathrm{CH}_{3}\right), 21.4\left(\mathrm{CH}_{3}\right), 23.1(\mathrm{Cl}), 26.0\left(\mathrm{H}_{2} \mathrm{C} 5\right), 27.6\left(\mathrm{CH}_{3}\right), 31.4\left(\mathrm{H}_{2} \mathrm{C} 5\right), 35.2$ $\left(\mathrm{HC}\left(\mathrm{CH}_{3}\right)_{2}\right), 115.8(\mathrm{HC} 3), 139.9(\mathrm{C} 4)$.

1-Methyl-4-(1'-methylcyclopropyl)-7-oxabicyclo[4.1.0]heptane (12). Obtained as cis/trans 31/69 diastereomeric mixture.Yield : $97 \% ; \mathrm{m} / \mathrm{z}: 167\left(8.20 \%, \mathrm{MH}^{+}\right) ;{ }^{1} \mathrm{H} \mathrm{NMR}\left(\mathrm{CDCl}_{3}\right): \delta=$ $0.10(\mathrm{br} \mathrm{s}, 4 \mathrm{H}), 0.79(\mathrm{~s}, 3 \mathrm{H}), 1.20(\mathrm{~s}, 3 \mathrm{H}), 1.41-1.99(\mathrm{~m}, 7 \mathrm{H}), 2.88(\mathrm{~d}, \mathrm{~J}=5.4 \mathrm{~Hz}, 0.7 \mathrm{H}$, trans diastereoisomer), 2.95 (br s, $0.3 \mathrm{H}$, cis diastereoisomer); ${ }^{13} \mathrm{C} \mathrm{NMR}\left(\mathrm{CDCl}_{3}\right): \delta=12.4$ and 12.5 $\left(\mathrm{H}_{2} \mathrm{C}^{\prime}\right.$ ', $\left.\mathrm{H}_{2} \mathrm{C}^{\prime}{ }^{\prime}\right), 18.3$ and $18.5\left(\mathrm{Cl}^{\prime}\right), 18.8$ and $18.9\left(\mathrm{CH}_{3}\right), 23.1$ and $25.6\left(\mathrm{H}_{2} \mathrm{C} 3\right), 23.2$ and 24.6 $\left(\mathrm{CH}_{3}\right), 27.6$ and $29.5\left(\mathrm{H}_{2} \mathrm{C} 5\right), 29.1$ and $31.1\left(\mathrm{H}_{2} \mathrm{C} 2\right), 37.3$ and $41.7(\mathrm{HC} 4), 57.6$ and $57.8(\mathrm{C} 1)$, 59.6 and 61.3 (HC6).

Oxidative cleavage (general procedure). In a typical procedure, water $(6 \mathrm{~mL})$, periodic acid $(5.67 \mathrm{~g}, 24.8 \mathrm{mmol})$ and $\mathrm{NaOH}(0.99 \mathrm{~g}, 24.8 \mathrm{mmol})$ were introduced in a three necked flask equipped with a magnetic stirrer bar. The mixture was stirred and cooled at $0{ }^{\circ} \mathrm{C}$ (ice bath). Then, at this temperature were successively added $\mathrm{CCl}_{4}(4 \mathrm{~mL}), \mathrm{CH}_{3} \mathrm{CN}(4 \mathrm{~mL})$ and $\mathrm{RuCl}_{3} \times 3 \mathrm{H}_{2} \mathrm{O}$ $(0.0165 \mathrm{~g}, 0.063 \mathrm{mmol})$. After $15 \mathrm{~min}$, the substrate $(0.422 \mathrm{~g}, 3.1 \mathrm{mmol})$ was added. The ice bath 
was removed and the reaction, conducted at room temperature, was monitored by gas chromatography of samples taken at regular time intervals. At the end of the reaction, $25 \mathrm{~mL}$ of $\mathrm{CHCl}_{3}$ were added, and the organic layer was washed, dried $\left(\mathrm{MgSO}_{4}\right)$, filtered on silica gel in order to remove the precipitated $\mathrm{RuO}_{4}$ and then concentrated to give the corresponding cyclopropyl-ketoacids.

[2,2-Dimethyl-1-(3'-oxobutyl)-cyclopropyl]-acetic acid (13). Yield : $81 \%$; mp 94-95 ${ }^{\circ} \mathrm{C}$ (hexane / ether 1:1); m/z : $\left.199(55.74 \%, \mathrm{MH}\rceil^{+}\right) ;{ }^{1} \mathrm{H}$ NMR $\left(\mathrm{CDCl}_{3}\right): \delta=0.20(\mathrm{~d}, \mathrm{~J}=4.4 \mathrm{~Hz}, 1 \mathrm{H})$, $0.25(\mathrm{~d}, \mathrm{~J}=4.4 \mathrm{~Hz}, 1 \mathrm{H}), 1.07$ (s, 3H), 1.09 (s, 3H), $1.70(\mathrm{~m}, 2 \mathrm{H}), 2.09$ (s, 3H), 2.23 (m, 2H), 2.49 $(\mathrm{m}, 2 \mathrm{H}) ;{ }^{13} \mathrm{C} \mathrm{NMR}\left(\mathrm{CDCl}_{3}\right): \delta=15.1\left(\mathrm{CH}_{3}\right), 20.7(\mathrm{C} 2), 21.9\left(\mathrm{CH}_{3}\right), 24.6(\mathrm{C} 1), 25.6\left(\mathrm{H}_{2} \mathrm{C} 3\right), 27.2$ $\left(\mathrm{H}_{2} \mathrm{Cl}^{\prime}\right.$ '), $29.9\left(\mathrm{CH}_{3}\right), 37.5\left(\mathrm{H}_{2} \mathrm{C} 2^{\prime}\right), 41.5\left(\mathrm{H}_{2} \underline{\mathrm{C}}-\mathrm{COOH}\right), 178.2(\underline{\mathrm{COOH}}), 209.2\left(\mathrm{C}^{\prime}\right)$.

(Z) [1-Methyl-2-(3'-methyl-2'-oxobutyl)-cyclopropyl]-acetic acid (14). Yield : 78 \% ; viscous oil; $\mathrm{m} / \mathrm{z}: 199\left(75.64 \%, \mathrm{MH}^{+}\right) ;{ }^{1} \mathrm{H} \mathrm{NMR}\left(\mathrm{CDCl}_{3}\right): \delta=0.10(\mathrm{~m}, 2 \mathrm{H}), 0.59(\mathrm{~m}, 1 \mathrm{H}), 0.97(\mathrm{~s}, 3 \mathrm{H})$, $0.99(\mathrm{~s}, 3 \mathrm{H}), 1.06(\mathrm{~s}, 3 \mathrm{H}), 2.44(\mathrm{br} \mathrm{s}, 2 \mathrm{H}), 2.46-2.55(\mathrm{~m}, 3 \mathrm{H}) ;{ }^{13} \mathrm{C} \mathrm{NMR}\left(\mathrm{CDCl}_{3}\right): \delta=15.3\left(\mathrm{CH}_{3}\right)$, $18.2\left(\mathrm{H}_{2} \mathrm{C} 3\right), 18.7\left(\mathrm{CH}_{3}\right), 24.8\left(\mathrm{CH}_{3}\right), 33.6(\mathrm{HC} 2), 40.3\left(\mathrm{HC} 3{ }^{\prime}\right), 40.9\left(\mathrm{H}_{2} \underline{\mathrm{C}}-\mathrm{COOH}\right), 43.9(\mathrm{C} 1)$, $48.1\left(\mathrm{H}_{2} \mathrm{C} 1^{\prime}\right), 178.2(\mathrm{COOH}), 213.8\left(\mathrm{C}^{\prime}\right)$.

3-(1'-Methylcyclopropyl)-6-oxoheptanoic acid (15). Yield : 85 \%, visquous oil; m/z : 199 $\left(33.61 \%, \mathrm{MH}^{+}\right) ;{ }^{1} \mathrm{H} \mathrm{NMR}\left(\mathrm{CDCl}_{3}\right): \delta=0.25(\mathrm{~m}, 4 \mathrm{H}), 0.85(\mathrm{~s}, 3 \mathrm{H}), 1.63(\mathrm{q}, \mathrm{J}=7.8 \mathrm{~Hz}, 2 \mathrm{H})$, $2.09(\mathrm{~s}, 3 \mathrm{H}), 2.10-2.50(\mathrm{~m}, 5 \mathrm{H}) ;{ }^{13} \mathrm{C} \mathrm{NMR}\left(\mathrm{CDCl}_{3}\right): \delta=12.2\left(\mathrm{H}_{2} \mathrm{C} 2\right.$ ') $, 14.7\left(\mathrm{H}_{2} \mathrm{C} 3{ }^{\prime}\right), 17.7\left(\mathrm{CH}_{3}\right)$, 18.0 (C1'), $26.9\left(\mathrm{H}_{2} \mathrm{C} 4\right), 29.9\left(\mathrm{CH}_{3}\right), 38.4\left(\mathrm{H}_{2} \mathrm{C} 2\right), 41.7\left(\mathrm{H}_{2} \mathrm{C} 5\right), 43.0(\mathrm{HC} 3), 179.3(\mathrm{C} 1), 209.2$ (C6).

[2,2-Dichloro-3,3-dimethyl-1-(3'-oxobutyl)-cyclopropyl]-acetic acid (16). Yield : 79 \% ; 104$105{ }^{\circ} \mathrm{C}$ (hexane / ether 1:1); m/z : $\left.271(0.66 \%, \mathrm{MH}+4\rceil^{+}\right) ; 269\left(6.23 \%, \mathrm{MH}+27^{+}\right) ; 267(9.84 \%$, $\left.\mathrm{MH} 7^{+}\right) ;{ }^{1} \mathrm{H}$ NMR $\left(\mathrm{CDCl}_{3}\right): \delta=1.30(\mathrm{~s}, 3 \mathrm{H}), 1.32(\mathrm{~s}, 3 \mathrm{H}), 1.98(\mathrm{~m}, 2 \mathrm{H}), 2.18(\mathrm{~s}, 3 \mathrm{H}), 2.55(\mathrm{~m}$, 2H), $2.70(\mathrm{~m}, 2 \mathrm{H}) ;{ }^{13} \mathrm{C} \mathrm{NMR}\left(\mathrm{CDCl}_{3}\right): \delta=19.0\left(\mathrm{CH}_{3}\right), 20.1\left(\mathrm{CH}_{3}\right), 23.6\left(\mathrm{H}_{2} \mathrm{Cl}{ }^{\prime}\right), 30.0\left(\mathrm{CH}_{3}\right)$, $31.3(\mathrm{C} 2), 33.1(\mathrm{C} 1), 34.3\left(\mathrm{H}_{2} \underline{\mathrm{CCOOH}}\right), 40.1\left(\mathrm{H}_{2} \mathrm{C} 2 '\right), 75.4\left(\mathrm{Cl}_{2} \mathrm{C} 3\right), 176.7(\mathrm{COOH}), 208.0(\mathrm{C} 3)$.

(Z) [2,2-Dichloro-1-methyl-3-(3'-methyl-2'-oxobutyl)-cyclopropyl]-acetic acid (17). Yield : $83 \% ; 191-192{ }^{\circ} \mathrm{C}$ (hexane / ether $\left.\left.\left.1: 1\right) ; \mathrm{m} / \mathrm{z}: 271(9.84 \%, \mathrm{MH}+4\rceil^{+}\right) ; 269(54.10 \%, \mathrm{MH}+2\rceil^{+}\right)$; $267\left(100 \%, \mathrm{MH}^{\dagger}\right) ;{ }^{1} \mathrm{H} \mathrm{NMR}\left(\mathrm{CDCl}_{3}\right): \delta=1.06(\mathrm{~d}, \mathrm{~J}=6.9 \mathrm{~Hz}, 3 \mathrm{H}), 1.07(\mathrm{~d}, \mathrm{~J}=6.9 \mathrm{~Hz}, 3 \mathrm{H})$, $1.43(\mathrm{~s}, 3 \mathrm{H}), 1.69(\mathrm{t}, \mathrm{J}=6.9 \mathrm{~Hz}, 1 \mathrm{H}), 2.48(\mathrm{br} \mathrm{s}, 2 \mathrm{H}), 2.58-2.72(\mathrm{~m}, 3 \mathrm{H}) ;{ }^{13} \mathrm{C} \mathrm{NMR}\left(\mathrm{CDCl}_{3}\right): \delta=$ $18.1\left(\mathrm{CH}_{3}\right), 18.3\left(\mathrm{CH}_{3}\right), 22.1\left(\mathrm{CH}_{3}\right), 29.1(\mathrm{Cl}), 33.6(\mathrm{HC} 3), 36.4\left(\mathrm{H}_{2} \underline{\mathrm{CCOOH}}\right), 37.3\left(\mathrm{H}_{2} \mathrm{C} 1^{\prime}\right)$, 40.7 ( $\left.\mathrm{HC}^{\prime}\right), 69.8\left(\mathrm{Cl}_{2} \mathrm{C} 2\right), 176.5(\mathrm{COOH}), 211.9\left(\mathrm{C} 2{ }^{\prime}\right)$.

\section{References}

1. (a) Enayati, A. A.; Vatandoosty, H.; Ladonniy, H.; Townson, H.; Hemingway, J. Medical and Veterinary Entomology 2003, 17, 138-144. (b) Lynd, A.; Ranson, H.; McCall, P. J.; Randle, N. P.; Black IV, W. C.; Walker, E. D.; Donnelly, M. J. Malaria Journal 2005, 4:16, 1-5. (c) Takken, W. Tropical Medicine and International Health 2002, 7:12, 10221030. (d) Khambay, B. P. S. Pesticide Outlook 2002, 13(2), 49-54. (e) Bonnet, J.; Corbel, 
V.; Darriet, F.; Chandre, F.; Hougard, J. M. Journal of the American Mosquito Control Association 2004, 20 : 438-443.

2. (a) Gazzaeva, R.; Mochalov, S.; Archegov, B.; Zefirov, N. Chemistry of Heterocyclic Compounds 2005, 41, 272-272. (b) Gnad, F.; Rieser, O. Chem. Rev. 2003, 103, 1603-1623. (c) Pietruszka, J. Chem. Rev. 2003, 103, 1051-1070. (d) Bakunov, S. A.; Rukavishnikov, A. V.; Tkachev, A. V. Synthesis 1994, 935-938. (e) Lee, P. H.; Kim, J. S.; Kim, Y. C.; Kim, S. Tetrahedron Letters 1993, 34, 7583-7586. (f) Hammond, G. B.; Cox, M. B.; Wiemer, D. F. J. Org. Chem. 1990, 55, 128-132. (g) Arlt, D.; Jautelat, M.; Lantzsch, R. Angew. Chem. Int. Ed. Engl. 1981, 20, 703-722.

3. Ziyat, H. ; Ait Ali, M. ; Ait Itto, My Y.; Riahi; A.; Karim; A. ; Daron, J. C. Acta Cryst. 2002, C58, 90-93.

4. Ziyat, H.; El Houssame, S.; Ait Ali, M.; Ait Itto, My Y.; Karim, A.; Wartchow, R.; Butenschön, H. Z. Naturforsch. 2004, 59b, 1177-1179.

5. Ziyat, H.; Ait Ali, M. ; Karim, A. ; Meliet, C. ; Castanet, Y. ; Mortreux, A. Acta Chim. Slov. 2004, 51, 223-230.

6. (a) Wanga, M.-L.; Hsiehb, Y.-M.; Changb, R.-Y., React. Kinet. Catal. Lett. 2004, 81, 49-56.

(b) Dehmlow, E. V., Leffers, W., J. Organomet. Chem. 1985, 288, C41-C43. (c) Dehmlow, E. V., Prashad, M., J. Chem. Res. Synposes 1982, 354-355.

7. (a) Carlsen, J.; Katsuki, T.; Martin, V. S.; Sharpless, K. B. J. Org. Chem. 1981, 46, 39363938. (b) Petride, H.; Costan, O.; Drãghici, C.; Florea, C.; Petride, A. ARKIVOC 2005, (x), 18-32.

8. Jayachandran, P.; Wang, M.-L. Applied Catalysis A: General 2001, 206, 19-28.

9. (a) Wang, M.-L.; Hsieh, Y.-M.; Chang, R.-Y. Journal of Molecular Catalysis A: Chemical 2003, 198,111-124. (b) Sirovski, F.; Gorokhova, M.; Ruban, S. Journal of Molecular Catalysis A: Chemical 2003,197, 213-222.

10. Bachowska, B.; Zujewska, T. ARKIVOC 2001, (vi), 78-84.

11. (a) Juliá, S.; Ginebreda, A. Synthesis, 1977, 682. (b) Juliá, S.; Ginebreda, A. Ann. Quim. 1980, 76, 136.

12. Sirovski, F.; Reichardt, C.; Gorokhova, M.; Ruban, S.; Stoikova, E. Tetrahedron 1999, 55,6363-6374.

13. Makosza, M. Pure Appl. Chem. 1975, 43,439.

14. (a) El jamili, H.; Auhmani, A.; Dakir, M. ; Lassaba, E. ; Benharref, A. ; Pierrot, M. ; Chiaroni, A. ; Riche, C. Tetrahedron Lett. 2002, 43, 6645-6648. (b) H.; Auhmani, Kossabeva, E.; Lassaba, E. ; Reglier, M.; Pierrot, M. ; Benharref, A. Acta Cryst. 1999, C55, JUC 9900055.

15. (a) Makosza, M.; Ludwikow, M. Angew. Chem. Int. Ed. Engl. 1974, 13, 665-666. (b) Dehmlow, E. V.; Ezimora, G. C. Tetrahedron Lett. 1971, 12, 563-566.

16. Fdil, N.; Ait Ali, M.; Ait Itto, My Y.; Karim; A.; Daron, J. C. Tetrahedron Lett. 2002, 43, 8769-8771.

17. The cis/trans diastereomeric mixture was used in the next reaction without separation. 